CRC screening (direct measurement: adjusted odds ratio $(\mathrm{AOR})=1.26, \quad 95 \%$ confidence interval $(\mathrm{CI})=1.10-1.45$, $\mathrm{p}<0.001$; for indirect measurement: $\mathrm{AOR}=1.23$, $95 \%$ $\mathrm{CI}=1.08-1.41, \quad \mathrm{p}<0.01)$ were significantly associated with CRC screening uptake (table 2).

Conclusions The variables pertinent to the TPB could successfully predict the uptake of CRC screening. Promotion of CRC screening based on enhancing perceived behavioural control as well as behavioural intention could be effective in increasing screening uptake. Further studies are needed to establish a cause-and-effect relationship between these variables and uptake, as well as to evaluate the cost-effectiveness of such interventions.

\section{IDDF2018-ABS-0149 MANIPULATION OF GUT MICROBIOTA IN IN VITRO MODEL OF COLORECTAL CANCER: POSITIVE EFFECTS OF LACTOBACILLUS RHAMNOSUS AGAINST FUSOBACTERIUM NUCLEATUM}

Tan Wei Jian*, Norfilza Mohd Mokhtar, Raja Affendi Raja Ali, Wong Kon Ken. National University of Malaysia, Malaysia

\subsection{6/gutjnl-2018-IDDFabstracts. 130}

Background Colorectal cancer (CRC), a leading cause of morbidity and mortality worldwide, is affecting more than 1.36 million people every year. It is a well-known multifactorial disease, and the aetiology is a complex interaction between genetic, lifestyle and environmental factors. However, there has been a general consensus exists that the composition of the human intestinal microbiota is linked to health status. They mainly play a role in modulating normal gut immune function, and most of them are largely beneficial to human. Fusobacterium nucleatum is an anaerobic oral commensal and a periodontal pathogen associated with a wide spectrum of human diseases. Findings from human and animal models have revealed that fusobacterium nucleatum plays a role in colorectal carcinogenesis by suppressing the immune response of the hosts to tumour. The aim of the present study is to determine the role of a probiotic strain in competing with a selected colorectal cancer pathogen.

Methods Thus, lactobacillus rhamnosus was investigated in vitro to examine its ability to protect, displace and compete with fusobacterium nucleatum. In this study, three different types of assay (protection assay, displacement assay and competitive assay) were performed using SW480, a colorectal cancer cell line as a host cell. Cell proliferation assay was applied to determine the proliferation rate of the cells by measuring the cell metabolic activity.

Results In a competitive assay, lactobacillus rhamnosus showed greater ability of adherence to host cells than fusobacterium nucleatum with a ratio of 1400:1. Lactobacillus rhamnosus could significantly decrease the proliferation rate induced by fusobacterium nucleatum in protection assay $(\mathrm{p}<0.001)$. In addition, the proliferation rate induced by fusobacterium nucleatum was also decreased up to $48.6 \%$ in displacement assay by lactobacillus rhamnosus.

Conclusions This finding may suggest that lactobacillus rhamnosus could help limiting cell proliferation of the cancerous cells in response to pathogens. In conclusion, lactobacillus rhamnosus has a positive impact in reducing the risk of colorectal cancer and possibly an option for preventive.

\section{IDDF2018-ABS-0152 THE PREOPERATIVE CEA CAN BE AN INDEPENDENT PREDICTIVE FACTOR FOR COLORECTAL CANCER PATIENTS AFTER CURATIVE RESECTION IN LONG-TERM OUTCOME}

Shu Huan Huang*, Wen Sy Tsai, Jeng Fu You, Yu Jen Hsu, Yih Jong Chern, Hsin Yuan Hung, Chien Yuh Yeh, Pao Shiu Hsieh, Sum Fu Chiang, Cheng Chou Lai, Reiping Tang, Jinn Shiun Chen, Jy Ming Chiang. Linkou Chang Gung Memorial Hospital, Taiwan

\subsection{6/gutjnl-2018-IDDFabstracts. 131}

Background The preoperative carcinoembryonic antigen (CEA) level as a prognostic factor of colorectal cancer (CRC) has been in debate.

Methods A consecutive case series were collected from our database of CRC from 1995 to 2010. Clinicopathological factors were matched by propensity score methods with calliper width of 0.05 and a 1:4 ratio. Long-term overall survival (OS) and disease-specific survival (DSS) were analysed and compared between 3 different serum CEA levels.

Results A total number of 9634 patients were included initially. After exclusion and matching, a number of 6099 cases were matched by propensity score matching methods with median follow-up period 86 months.

The survival analysis showed poor OS in elevated serum CEA groups than normal CEA level (5 year survival rate: $75 \%$ vs. $66 \%$ vs. $61 \%$ ) and was also presented in both subgroups divided by lymph node (LN) stage (LN positive: $69 \%$ vs. $56 \%$ vs. $50 \%$, LN negative: $83 \%$ vs. $77 \%$ vs. $72 \%$ ) but not between elevated groups. In contrast, DSS showed significantly poor outcome between each group and was proportional to serum CEA elevation (5 year survival rate: $83 \%$ vs. $76 \%$ vs. $69 \%, \mathrm{p}<0.03$ between each group) and in LN negative subgroup (5 year survival rate: $92 \%$ vs. $87 \%$ vs. $82 \%$ ). In contrast, the elevated CEA in LN positive subgroup showed poor DSS (5 year survival rate: $75 \%$ vs. $65 \%$ vs. $56 \%$ ) than non-elevated group $(\mathrm{p}<0.001)$ but not between elevated groups $(\mathrm{p}=0.064)$.

In multivariate Cox regression model after adjusted with TNM stage, smoking, DM, renal insufficiency, liver cirrhosis and other factors, elevated CEA showed higher risk in DSS (5 to $10 \mathrm{ng} / \mathrm{ml} \mathrm{HR}=1.540,>10 \mathrm{ng} / \mathrm{ml} \mathrm{HR}=1.910$, both $\mathrm{p}<0.001)$. Conclusions Elevated preoperative serum CEA can be an independent prognostic factor for stage I to III CRC patients after curative resection with poor long-term overall and oncological outcome.

\section{IDDF2018-ABS-0158 ENDOSCOPIC PREDICTORS OF THE DEPTH INFILTRATION OF COLORECTAL LATERALLY SPREADING TUMOURS}

Zilin Kan, Peng Jin. PLA Army General Hospital, China

10.1136/gutjnl-2018-IDDFabstracts.132 